\title{
GROWTH-INDUCED INSTABILITIES OF AN ELASTIC FILM ON A VISCOELASTIC SUBSTRATE: ANALYTICAL SOLUTION AND COMPUTATIONAL APPROACH VIA EIGENVALUE ANALYSIS
}

\author{
Iman VAlizadeh, PAUl Steinmann And Ali JAVili
}

\begin{abstract}
The objective of this contribution is to study for the first time the growth-induced instabilities of an elastic film on a viscoelastic substrate using an analytical approach as well as computational simulations via eigenvalue analysis. The growth-induced instabilities of a thin film on a substrate is of particular interest in modeling living tissues such as skin, brain, and airways. The analytical solution is based on Airy's stress function adopted to viscoelastic constitutive behavior. The computational simulations, on the other hand, are carried out using the finite deformation continuum theory accounting for growth via the multiplicative decomposition of the deformation gradient into elastic and growth parts. To capture the critical growth of elastic films and the associated folding pattern, eigenvalue analysis is utilized, in contrast to the commonly used perturbation strategy. The eigenvalue analysis provides accurate, reliable, and reproducible solutions as contrasted to the perturbation approach. The numerical results obtained from the finite element method show an excellent agreement between the computational simulations and the proposed analytical solution.
\end{abstract}

\section{Introduction}

Instabilities of bilayered structures consisting of a thin stiff film adhered to an infinite substrate are increasingly important due to their applications in biological tissues. Such structural instabilities in the form of wrinkles [Cao and Hutchinson 2012b; Budday et al. 2014], folds [Sun et al. 2012; Sultan and Boudaoud 2008], or creases [Cao and Hutchinson 2012a; Hong et al. 2009; Jin et al. 2015] have been studied recently. In many living systems, the formation of structural instabilities is critical to appropriate biological function of the system [Wyczalkowski et al. 2012]. Typical examples are wrinkling of skin [Tepole et al. 2011], villi formation in the intestine [Balbi and Ciarletta 2013], and folding of the developing brain [Xu et al. 2010; Budday et al. 2014; Budday and Steinmann 2018]. However, in some biological systems, the formation of structural instabilities can be an indication of a disease, e.g., the folding of the mucous membrane in asthmatic airways [Wiggs et al. 1997].

It is thus not surprising that the mathematical modeling of folding in tubular organs [Ciarletta and Ben Amar 2012], in particular the modeling of the folding mucous membrane [Moulton and Goriely 2011; Li et al. 2011; Xie et al. 2014], has attracted increasing scientific attention in the past decade. This problem (and variants thereof) has been widely studied lately [Budday et al. 2015; Cao and Hutchinson 2012b; Huang et al. 2005; Hutchinson 2013; Jin et al. 2011; Sun et al. 2012; Xu et al. 2014].

The concept of growth commonly has been modeled by continuum approaches via the multiplicative decomposition of the deformation gradient into an elastic and a growth part [Rodriguez et al. 1994] which

Keywords: growth-induced instabilities, viscoelasticity, wrinkling, finite element method. 
pairs the growth to the kinematic level [Taber 1995]. This concept requires the introduction of an artificial intermediate configuration [Garikipati et al. 2004]. Additional details on the continuum theory of growth and its implications are discussed in [Ciarletta and Maugin 2011; Ciarletta et al. 2013; Dervaux and Ben Amar 2011; Dunlop et al. 2010; Epstein and Maugin 2000; Garikipati et al. 2004; Goriely et al. 2008; Kuhl et al. 2003; Li et al. 2011; Yavari 2010; Ben Amar and Goriely 2005; Javili et al. 2014], amongst others. Growth is commonly formulated within the framework of open-system thermodynamics [Kuhl and Steinmann 2003; Kuhl 2014] where the body is allowed to constantly exchange mass, momentum, and entropy with its environment through corresponding fluxes across its boundary; see also [Cowin and Hegedus 1976; Epstein and Maugin 2000; Javili et al. 2013].

Most of the contributions on the subject assume the compliant substrate to be elastic. This contribution for the first time studies the growth-induced instabilities of an elastic film on a viscoelastic substrate from both analytical and computational perspectives using eigenvalue analysis and, in particular, elaborates on the role of the relaxation time on the instability pattern as well as the critical growth; see also [Huang and Suo 2002; Huang 2005; Budday et al. 2014]. Key features of this contribution are:

(1) to study the growth-induced instabilities of an elastic film on a viscoelastic substrate from both analytical and numerical perspectives,

(2) to employ the eigenvalue analysis proposed in [Javili et al. 2015] for the numerical solution and not the common perturbation strategy, and

(3) to illustrate an excellent agreement between the numerical and analytical solutions.

This manuscript is organized as follows. Section 2 deals with the computational approach to study growth-induced instabilities of a thin film on a compliant viscoelastic substrate. Next, the analytical solution of the problem is derived in Section 3 and its simplification to various classes of viscoelastic models are discussed. The results from the computational approach using the finite element method are compared against the analytical solution through a series of numerical examples in Section 4 and it is found that the two strategies are in excellent agreement. Finally, Section 5 concludes this work and provides further outlook.

\section{Computational approach}

The numerical solution of the problem is achieved by using the finite deformation theory in continuum mechanics to account for growth, whereby the deformation gradient is decomposed multiplicatively into an elastic and a growth part.

Let the continuum body $\mathcal{B}_{0}$ occupy the material configuration at time $t=0$, as shown in Figure 1 . The motion $\varphi$ maps the body $\mathcal{B}_{0}$ to the spatial configuration $\mathcal{B}_{t}$ at time $t$. The deformation gradient $\boldsymbol{F}$ maps the line element $\mathrm{d} \boldsymbol{x}$ from $\mathcal{B}_{0}$ to $\mathrm{d} \boldsymbol{x}$ in $\mathcal{B}_{t}$ and is defined as $\boldsymbol{F}:=\operatorname{Grad} \boldsymbol{\varphi}$. The governing balance equations of finite deformation continuum mechanics consist of the balance of linear and angular momentum. The balance of linear momentum in material configuration, for body $\mathcal{B}_{0}$ at time $t=0$ and a quasistatic process, reads

$$
\operatorname{Div} \boldsymbol{P}+\boldsymbol{b}_{0}=\mathbf{0} \quad \text { in } \mathcal{B}_{0} \text { subject to } \boldsymbol{t}=\boldsymbol{t}_{0} \text { on } \partial \mathcal{B}_{0} \text { with } \boldsymbol{t}=\boldsymbol{P} \cdot \boldsymbol{N},
$$



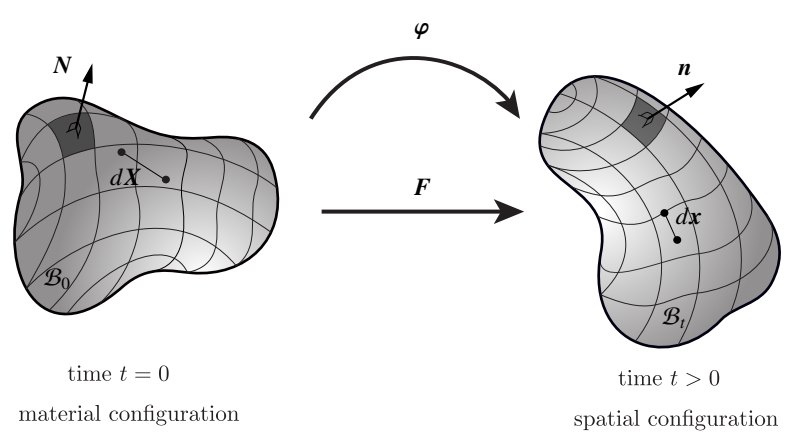

Figure 1. The material and spatial configurations of a continuum body with associated nonlinear deformation map $\boldsymbol{\varphi}$ and the linear tangent map $\boldsymbol{F}:=\operatorname{Grad} \boldsymbol{\varphi}$.

where $\boldsymbol{P}$ is Piola stress ${ }^{1}$ and $\boldsymbol{b}_{0}$ is the body force density in the material configuration. The balance of angular momentum leads to the symmetry of the Cauchy stress tensor $\sigma=\sigma^{\mathrm{t}}$ related to the Piola stress via $\boldsymbol{P}=\boldsymbol{\sigma} \cdot \operatorname{Cof} \boldsymbol{F}$.

2.1. Growing elastic film. To model volumetric growth one can use the multiplicative decomposition of the deformation gradient $\boldsymbol{F}$ into a growth part $\boldsymbol{F}_{g}$ and an elastic part $\boldsymbol{F}_{e}$ as

$$
\boldsymbol{F}=\boldsymbol{F}_{e} \cdot \boldsymbol{F}_{g} \Rightarrow \boldsymbol{F}_{e}=\boldsymbol{F} \cdot \boldsymbol{F}_{g}^{-1} \quad \text { and } \quad J=J_{e} J_{g}, \quad J_{e}=\operatorname{det} \boldsymbol{F}_{e}, \quad J_{g}=\operatorname{det} \boldsymbol{F}_{g},
$$

where $J$ is the Jacobian determinant of $\boldsymbol{F}$ and indicates the volume change due to the deformation as $J=$ $\mathrm{d} v / \mathrm{d} V$. In modeling growth, the growth part $\boldsymbol{F}_{g}$ maps the body $\mathcal{B}_{0}$ from the material configuration to an intermediate stress-free "configuration", which may be incompatible. The elastic part of the deformation gradient $\boldsymbol{F}_{e}$ maps the intermediate "configuration" to the compatible spatial configuration as shown in Figure 2. Here, growth is assumed to be morphogenetic and thus independent of the deformation itself. We consider anisotropic growth along the film such that it prevents growth in the lateral directions. Hence, the growth tensor can be described as $\boldsymbol{F}_{g}=\boldsymbol{I}+g \boldsymbol{I}_{\mathrm{ani}}$, where $\boldsymbol{I}_{\text {ani }}=\boldsymbol{I}-\boldsymbol{N} \otimes \boldsymbol{N}$ with $\boldsymbol{N}$ being the unit normal vector to the film. Note that in the absence of growth, the growth tensor $\boldsymbol{F}_{g}=\boldsymbol{I}$ and the deformation gradient $\boldsymbol{F}$ is equal to the elastic part. The growth parameter $g$ represents growth if $g>0$ and shrinkage or atrophy if $g<0$.

The constitutive behavior of the film is identified via its free energy $\psi$ depending on the growth part of the deformation gradient $\boldsymbol{F}_{g}$ and $\boldsymbol{F}$, respectively. Therefore, the free energy $\psi\left(\boldsymbol{F}, \boldsymbol{F}_{g}\right)$ renders the same value as the elastic free energy $\psi_{e}\left(\boldsymbol{F}_{e}\right)$ as

$$
\psi=\psi\left(\boldsymbol{F}, \boldsymbol{F}_{g}\right)=\psi_{e}\left(\boldsymbol{F}_{e}\right)
$$

\footnotetext{
${ }^{1}$ The term Piola stress is adopted instead of the more commonly used first Piola-Kirchhoff stress. Nonetheless, it seems that the term Piola stress is more appropriate for this stress measure. Recall, $\boldsymbol{P}$ is essentially the Piola transform of the Cauchy stress and ties perfectly to the Piola identity. Also historically, Kirchhoff (1824-1877) employed this stress measure after Piola (1794-1850); see also the discussion in [Podio-Guidugli 2000].
} 


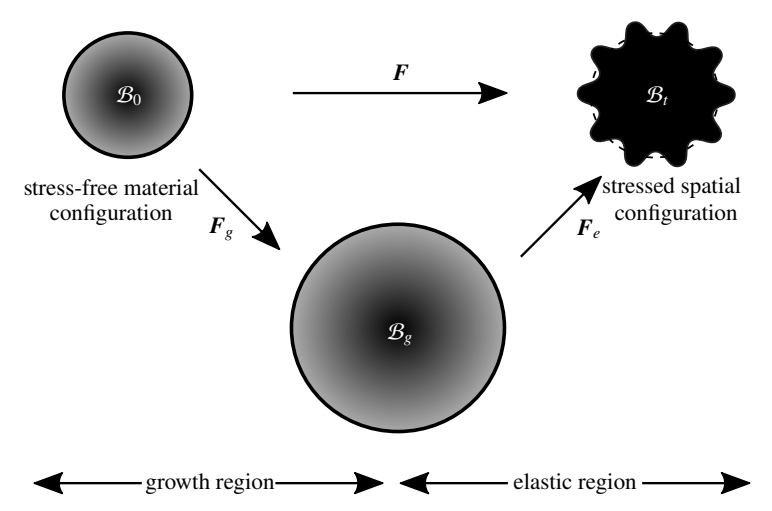

Figure 2. Kinematics of growth with multiplicative decomposition of the deformation gradient into elastic $\boldsymbol{F}_{e}$ and growth $\boldsymbol{F}_{g}$ parts. The intermediate configuration $\mathcal{B}_{g}$ is, in general, incompatible.
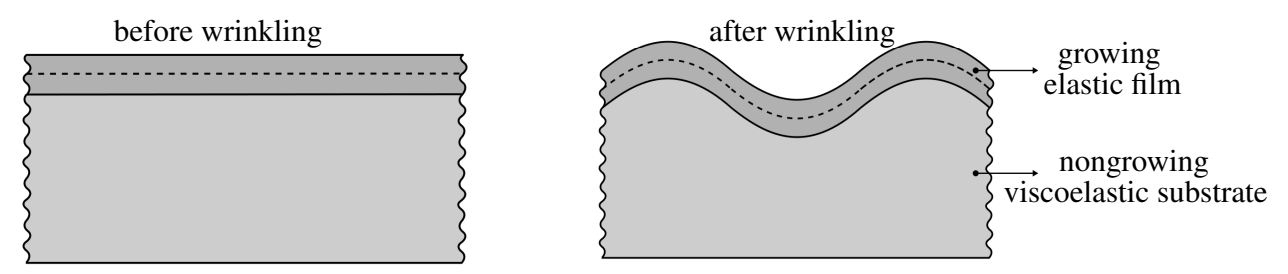

Figure 3. Geometry of elastic growing film on a viscoelastic substrate.

Due to the second law of thermodynamics and using the Coleman-Noll procedure, the Piola stress for a hyperelastic material reads

$$
\boldsymbol{P}:=\frac{\partial \psi}{\partial \boldsymbol{F}}=\frac{\partial \psi_{e}}{\partial \boldsymbol{F}_{e}}: \frac{\partial \boldsymbol{F}_{e}}{\partial \boldsymbol{F}}=\frac{\partial \psi_{e}}{\partial \boldsymbol{F}_{e}}:\left[\boldsymbol{I} \bar{\otimes} \boldsymbol{F}_{g}^{-\mathrm{t}}\right]=\boldsymbol{P}_{e} \cdot \boldsymbol{F}_{g}^{-\mathrm{t}} \quad \text { with } \quad \boldsymbol{P}_{e}:=\frac{\partial \psi_{e}}{\partial \boldsymbol{F}_{e}},
$$

where the operator $\bar{\otimes}$ denotes a nonstandard dyadic product with the index notation property $[\boldsymbol{A} \bar{\otimes} \boldsymbol{B}]_{i j k l}=$ $[\boldsymbol{A}]_{i k}[\boldsymbol{B}]_{j l}$ for two second-order tensors $\boldsymbol{A}$ and $\boldsymbol{B}$.

2.2. Viscoelastic substrate. As the film grows, the stress in the film increases until the growth parameter reaches a critical value $g_{c}$ at which point geometrical instabilities may occur in the form of wrinkles. Obviously, the corresponding deformed state is strongly dependent on the substrate beneath the film and thus the material behavior of the substrate plays an important role. In the problem of interest here, we consider an elastic growing film on a viscoelastic substrate as illustrated in Figure 3. The two sides are constrained in the horizontal direction and the bottom of the substrate is constrained in the vertical direction. The interface between the film and substrate is perfect and no debonding nor separation occurs throughout the process. This generalization shall be investigated in a future contribution.

The viscoelastic behavior of the substrate can be captured by introducing internal variables. For simplification, we consider the process to be isothermal and therefore neglect any temperature effects. Hence, the thermodynamic state of the body can be expressed merely by the deformation gradient and the internal variables. The free energy representing the viscoelastic material behavior of the substrate is 
based on an additive decomposition of the energy into its volumetric and isochoric parts together with a dissipative contribution $\psi^{\text {dis }}$ incorporating internal variables $\boldsymbol{\alpha}$ as

$$
\psi(J, \overline{\boldsymbol{C}})=\psi^{\mathrm{vol}}(J)+\psi^{\mathrm{iso}}(\overline{\boldsymbol{C}})+\psi^{\mathrm{dis}}(\boldsymbol{\alpha}, \overline{\boldsymbol{C}}) \quad \text { with } \quad \overline{\boldsymbol{C}}=J^{-2 / 3} \boldsymbol{C}, \boldsymbol{C}=\boldsymbol{F}^{\mathrm{t}} \cdot \boldsymbol{F} .
$$

The Piola-Kirchhoff stress $\boldsymbol{S}$ can be determined as

$$
\boldsymbol{S}=\boldsymbol{S}^{\mathrm{vol}}+\boldsymbol{S}^{\text {iso }}+\boldsymbol{Q} \quad \text { with } \quad \boldsymbol{S}^{\mathrm{vol}}=2 \frac{\partial \psi^{\mathrm{vol}}}{\partial \boldsymbol{C}}, \boldsymbol{S}^{\text {iso }}=2 \frac{\partial \psi^{\text {iso }}}{\partial \boldsymbol{C}}, \text { and } \boldsymbol{Q}=2 \frac{\partial \psi^{\mathrm{dis}}(\boldsymbol{\alpha}, \overline{\boldsymbol{C}})}{\partial \boldsymbol{C}},
$$

from which the Piola stress is readily obtained by $\boldsymbol{P}=\boldsymbol{F} \cdot \boldsymbol{S}$. The evolution equation for $\boldsymbol{Q}$ is assumed as

$$
\dot{\boldsymbol{Q}}+\frac{1}{\tau} \boldsymbol{Q}=\frac{1}{\tau} \dot{\boldsymbol{S}}_{\text {iso }},
$$

where $\tau$ denotes the relaxation time with the definition

$$
\tau=\frac{\eta}{E}
$$

in which $\eta$ and $E$ are the viscosity and the elastic modulus of the material, respectively. For $Q$ the convolution representation, as proposed in [Holzapfel 2000; Simo and Hughes 1998], reads

$$
\boldsymbol{Q}=\exp \left(-\frac{T}{\tau}\right) \boldsymbol{Q}+\int_{t=0}^{t=T} \frac{1}{\tau} \exp \left(-\frac{T-t}{\tau}\right) \dot{\boldsymbol{S}}_{\text {iso }} \mathrm{d} t
$$

\section{Analytical approach}

Since we are only interested in the onset of instabilities, unlike the computational approach to this problem, the analytical solution is derived based on small strains instead of finite deformations. In the computational approach, it would be impossible to capture instabilities if geometrical nonlinearities were precluded. Nonetheless, the geometrical instabilities in the analytical approach are implicitly accounted for via a buckling analysis of the film. To study the viscoelastic behavior of the substrate at small strains, we choose a rheological model demonstrated in Figure 4 representing the (general) standard solid model to recover a wide range of material behaviors; see [Holzapfel 2000; Simo and Hughes 1998] for further details. As it will be clarified, the (general) standard solid model captures both the Maxwell model and the Kelvin model. The rheological model in Figure 4 consists of two spring elements with constants $E$ and $E_{\infty}$, which represent the elastic response of the solid. The spring with constant $E$ is connected in series with a dashpot with viscosity $\eta$. From a physical point of view, the constants $E_{\infty}, E$, and $\eta$ must be positive. The strains in both elements A and B are identical due to their parallel arrangement. The total stress $\sigma$ prescribed in Figure 4 can be recovered as addition of the stress in A and B as

$$
\sigma=\sigma^{\infty}+\sigma^{\nu} \text { with } \sigma^{\infty}=E_{\infty} \boldsymbol{\varepsilon}
$$

where $\sigma^{\infty}$ is applied to the element B representing the stress of the rheological model as $t \rightarrow \infty$ in a relaxation test and $\sigma^{v}$ represents the viscous stress acting on the dashpot. From a mechanical point of view, the strain in the viscous element $B$ is the addition of the elastic strain in the spring with constant $E$ and the inelastic strain-like internal variable $\alpha$ in the dashpot and thus

$$
\boldsymbol{\sigma}^{\nu}=E[\varepsilon-\alpha]=\eta \dot{\alpha} .
$$




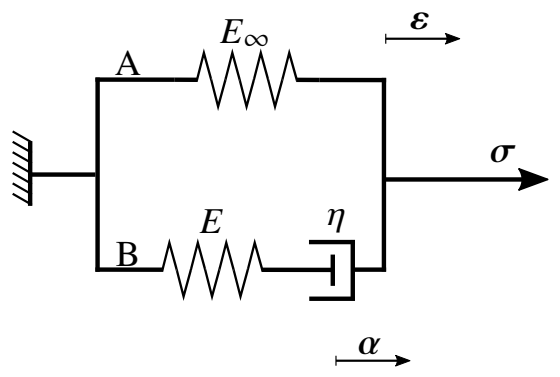

Figure 4. Illustration of the kinematics of the viscoelastic material with (general) standard solid element model. The rheological model is composed of two elements A and B. The subscript $\infty$ denotes the elastic response of the solid for $t \rightarrow \infty$ corresponding to the behavior of solid in a relaxation test after infinite time.

By considering the initial modulus $E_{0}$ at $t=0$ with no strain in the dashpot, the rheological model in Figure 4 resembles a solid with two spring elements with constants $E$ and $E_{\infty}$ as instantaneous modulus $E_{0}=E_{\infty}+E$ and hence, (10) leads to

$$
\boldsymbol{\sigma}=E_{0} \boldsymbol{\varepsilon}-E \boldsymbol{\alpha},
$$

where the inelastic strain $\alpha$ satisfies the evolution equation

$$
\dot{\boldsymbol{\alpha}}+\frac{1}{\tau} \boldsymbol{\alpha}=\frac{1}{\tau} \boldsymbol{\varepsilon} \quad \text { with the condition } \quad \lim _{t \rightarrow-\infty} \boldsymbol{\alpha}(t)=0 .
$$

Additionally, there exists an alternative formulation by introducing a stress-like variable $\boldsymbol{q}=E[\boldsymbol{\varepsilon}-\boldsymbol{\alpha}]$ acting on the dashpot, so that (12) transforms to $\sigma=E_{\infty} \boldsymbol{\varepsilon}+\boldsymbol{q}$ and thus the evolution equation (13) can be rewritten as

$$
\dot{\boldsymbol{q}}+\frac{1}{\tau} \boldsymbol{q}=\frac{1}{\tau} E \boldsymbol{\varepsilon} \quad \text { with the condition } \quad \lim _{t \rightarrow-\infty} \boldsymbol{q}(t)=\mathbf{0} .
$$

To determine the critical growth of an elastic film on a viscoelastic substrate, we compute the critical growth by analyzing the buckling of the film on the substrate, Figure 5. In doing so, first we assume the substrate to be elastic and then we replace the elastic behavior of the substrate by its equivalent viscoelastic one. The whole analysis here is two-dimensional and corresponding to a plane-strain scenario. Let $w$ denote the deflection of the film. The governing differential equation of a film adhered to an infinite half-space reads

$$
\frac{1}{12} E_{f} h^{3} \frac{\mathrm{d}^{4} w}{\mathrm{~d} x^{4}}+h \sigma \frac{\mathrm{d}^{2} w}{\mathrm{~d} x^{2}}=f_{s},
$$

where $\sigma$ is the stress in the film, $E_{f}$ is the film elastic modulus and $h$ is the film thickness. The transverse force on the film from the substrate $f_{s}$ reads [Allen 1969]

$$
f_{s}=-\frac{2 E_{s}}{\left[3-v_{s}\right]\left[1+v_{s}\right]} n w,
$$




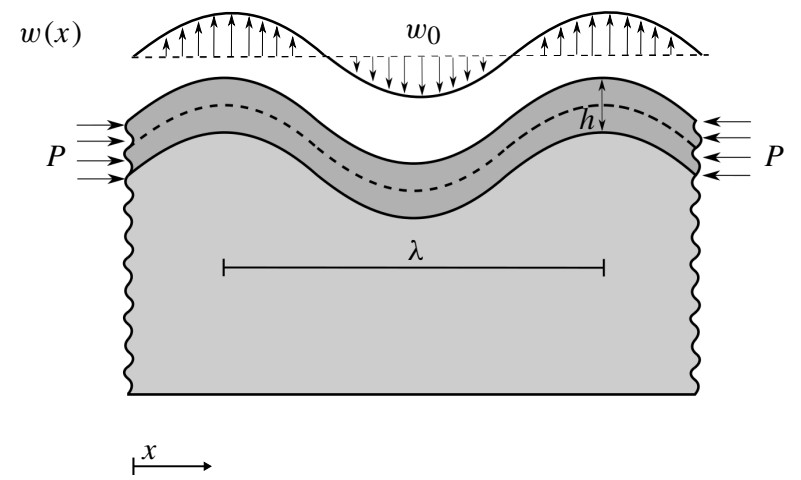

Figure 5. Analytical model of growing elastic film on a viscoelastic substrate. The film thickness is denoted $h$ and $\lambda$ is the wavelength. The amplitude of the sinusoidal wave on the substrate is denoted $w_{0}$. The lateral force $P$ relates to the stress in the film via $P=\sigma h b$ with $b$ being the width of the domain in the direction normal to the plane.

where $v_{s}$ is the Poisson's ratio of the substrate, with sinusoid $w$ with the wavenumber $n$ on its surface. By substituting $f_{s}$ in (15) and solving for $\sigma$, we have

$$
\sigma=\frac{1}{12} E_{f} h^{2} n^{2}+\frac{2 E_{s}}{\left[3-v_{s}\right]\left[1+v_{s}\right] h n},
$$

from which the critical wavenumber can be computed by minimizing with respect to $\sigma$ as

$$
n_{c}=\sqrt[3]{\frac{12 E_{s}}{E_{f}\left[3-v_{s}\right]\left[1+v_{s}\right] h^{3}}},
$$

from which the critical wavelength can be readily calculated. Inserting the critical wavenumber $n_{c}$ into the stress equation (17) results in the critical stress $\sigma_{c}$ and eventually the critical growth $g_{c}$ is obtained as $g_{c} \approx \varepsilon_{c}=\sigma_{c} / E_{f}$ for sufficiently small values of $\varepsilon_{c}$. A more accurate approximation for the critical growth reads $g_{c}=\varepsilon_{c} /\left[1-\varepsilon_{c}\right]$. Nonetheless, the validity of this linear approach is questionable for larger $\varepsilon_{c}$ corresponding to film-to-substrate stiffness ratios less than 10; see [Cao and Hutchinson 2012b].

Now, we generalize the elastic model to a viscoelastic one to study the effect of the viscoelastic material properties of the substrate on the critical growth of the film. To do so, by regarding $E_{s}$ and $v_{s}$ as the material constants of the substrate, the viscoelastic substrate model can be expressed solely by modifying the material constants of an elastic substrate model. First, by solving the internal variable in (14) in the linear viscoelastic regime, we have for the elastic modulus of the viscoelastic substrate

$$
E_{s}=E_{\infty}+E \exp \left(-\frac{\Delta t}{\tau}\right)
$$

It can be observed that for a large relaxation time $\tau$ compared to the growth time $\Delta t$, the substrate elastic modulus results in $E_{s}=E_{\infty}+E$, where the substrate can be viewed as an elastic substrate. On the other hand, for relatively slowly growing film or alternatively small relaxation time, the exponential term tends to 0 resulting in the effective elastic modulus in substrate $E_{s}=E_{\infty}$. To formulate this in the governing 

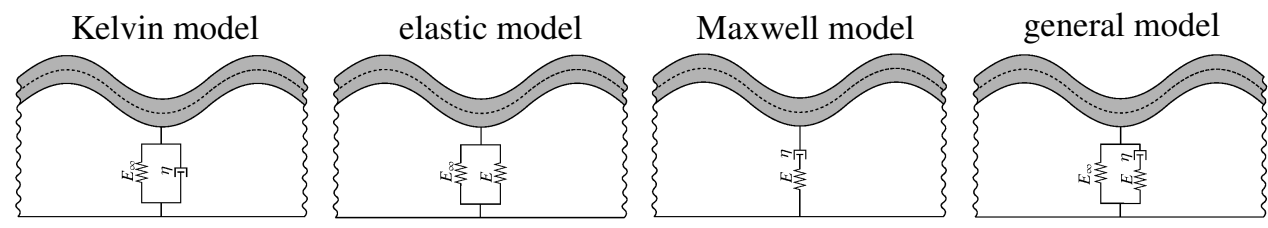

Figure 6. Illustration of four models for the substrate behavior. The standard solid model can recover Maxwell, Kelvin, and elastic models as three special cases.

equations of a growing film on a viscoelastic substrate, we introduce the effective substrate stiffness

$$
E_{s}^{\mathrm{eff}}=E_{\infty}+\left[E_{s}-E_{\infty}\right] \exp \left(-\frac{\Delta t}{\tau}\right),
$$

and then by substituting (20) in (16) we obtain the viscoelastic substrate transverse force as

$$
f_{s}=-\frac{2}{\left[3-v_{s}\right]\left[1+v_{s}\right]}\left[E_{\infty}+\left[E_{s}-E_{\infty}\right] \exp \left(-\frac{\Delta t}{\tau}\right)\right] n w(x),
$$

and after substituting in (15) and solving for $\sigma$ we have

$$
\sigma=\frac{1}{12} E_{f} h^{2} n^{2}+\frac{2}{\left[3-v_{s}\right]\left[1+v_{s}\right] h n}\left[E_{\infty}+\left[E_{s}-E_{\infty}\right] \exp \left(-\frac{\Delta t}{\tau}\right)\right],
$$

and consequently, the minimization with respect to $\sigma$ yields the critical wavenumber

$$
n_{c}=\sqrt[3]{\frac{12\left[E_{\infty}+\left[E_{s}-E_{\infty}\right] \exp (-\Delta t / \tau)\right]}{E_{f} h^{3}\left[3-v_{s}\right]\left[1+v_{s}\right]}},
$$

from which the critical stress $\sigma_{c}$ and eventually the critical growth $g_{c}$ can be calculated, as before.

Obviously, the standard solid model in viscoelastic material modeling can simplify to the Maxwell, Kelvin, and elastic models, as schematically illustrated in Figure 6. For instance, from Figure 6 it is obvious that a standard solid model reduces to the Maxwell model if $E_{\infty} \rightarrow 0$, and thus the effective stiffness of the substrate in this case reads

$$
E_{s}=E \exp \left(-\frac{\Delta t}{\tau}\right)
$$

and consequently

$$
f_{s}=-\frac{2}{\left[3-v_{s}\right]\left[1+v_{s}\right]}\left[E_{s} \exp \left(-\frac{\Delta t}{\tau}\right)\right] n w(x)
$$

\section{Numerical examples}

The purpose of this section is to illustrate the growth-induced instabilities in a bilayer system composed of a thin growing film on top of a viscoelastic substrate as shown in Figure 7. In particular, we study the influence of a viscoelastic substrate on the critical wavelength due to growth-induced buckling patterns as well as the critical growth. More importantly, the numerical results obtained from the computational simulations using the finite element method are compared against the proposed analytical solution.

For all examples, to omit further complexities in interpreting the results, it is assumed that the critical growth is reached at the same $\Delta t$ independent of the stiffness ratio. Therefore, the relaxation time $\tau$ 

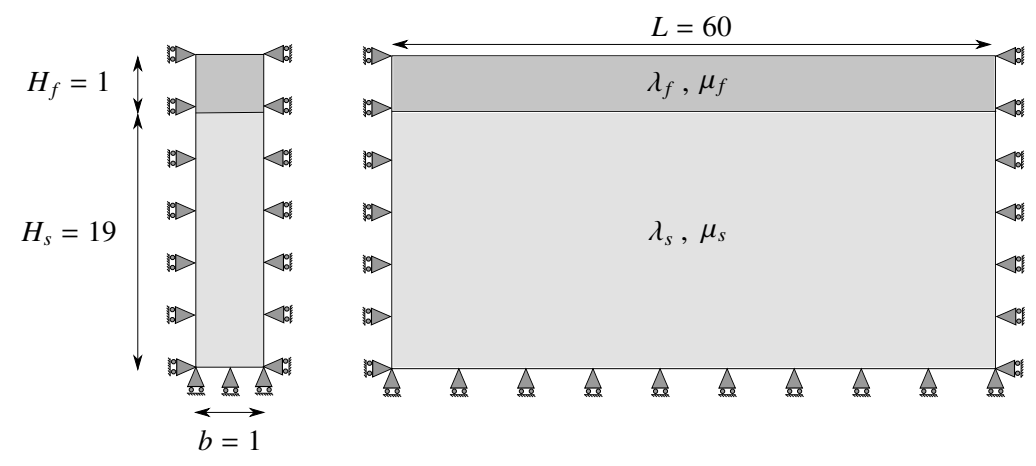

Figure 7. Geometry and dimensions of an elastic growing film on a viscoelastic substrate.

remains as the only independent parameter to study its effect instead of the ratio $\Delta t / \tau$. In order to extend the observations to a more general case with varying $\Delta t$, the dimensionless parameter $\kappa=\Delta t / \tau$ is defined, thereby $\kappa$ is essentially the ratio of the time for the film to reach the critical growth over the substrate relaxation time. For the problem of interest here, the neo-Hookean potential

$$
\psi_{e}=\frac{1}{2} \mu\left[\boldsymbol{F}_{e}: \boldsymbol{F}_{e}-2-2 \ln J_{e}\right]+\frac{1}{2} \lambda\left[\frac{1}{2}\left[J_{e}^{2}-1\right]-\ln J_{e}\right] \quad \text { with } \quad J_{e}=\operatorname{det} \boldsymbol{F}_{e}
$$

is used for the elastic response of the film and the substrate where $\mu$ and $\lambda$ are Lamé parameters. Furthermore, it is assumed that the film grows only along its length but not in the vertical direction. The film over substrate stiffness ratio is defined as $\mu_{f} / \mu_{s}$ and the Poisson's ratio for both media is assumed as $v_{f}=v_{s}=0.45$. Thus the larger the stiffness ratio, the more compliant is the substrate compared to the film. For the numerical simulations, the domain is discretized using biquadratic finite elements to achieve a better accuracy [Javili et al. 2015]. To compute critical growth of the film and folding pattern, the numerical approach based on the large deformation must be calculated. To this aim, first we weigh the strong form of the balance equations (1) with the test function $\delta \varphi \in \mathcal{H}_{0}^{1}\left(\mathcal{B}_{0}\right)$ with the definition $\delta \varphi=\mathbf{0}$ on $\delta \mathcal{B}_{0} \varphi$ and integrate over $\mathcal{B}_{0}$, yielding the following global weak form:

$$
\boldsymbol{r}^{\varphi}(\boldsymbol{\varphi}(\boldsymbol{x}, t))=\int_{\mathcal{B}_{0}} \operatorname{Grad} X \delta \boldsymbol{\varphi}: \boldsymbol{P} \mathrm{d} V-\int_{\mathcal{B}_{0}} \delta \boldsymbol{\varphi} \cdot \boldsymbol{b}_{0} \mathrm{~d} V-\int_{\partial \mathcal{B}_{0}} \delta \boldsymbol{\varphi} \cdot \boldsymbol{t}_{0} \mathrm{~d} A=\mathbf{0} .
$$

In the frame of finite element analysis, the goal is to solve (27) by vanishing the residual $r^{\varphi}(\varphi(x, t))$. To obtain this, and find $\varphi$ such that the residuum vanishes, the Newton-Raphson scheme can be used:

$$
\boldsymbol{r}\left(\boldsymbol{\varphi}_{m+1}\right)=\boldsymbol{r}\left(\boldsymbol{\varphi}_{m}\right)+\frac{\partial r}{\partial \boldsymbol{\varphi}} \cdot \Delta \boldsymbol{\varphi}
$$

where index $m$ denotes the iteration number. The derivative of the residual with respect to $\varphi$ can be described as the stiffness matrix $\boldsymbol{K}=\partial \boldsymbol{r} \partial \boldsymbol{\varphi}$ : the eigenvalue representation for diagonalizable matrices [Javili et al. 2015] of a stiffness matrix for a system with $n$ degrees of freedom is

$$
K_{n \times n}=K_{1} \lambda_{1} \otimes \lambda_{1}+K_{2} \lambda_{2} \otimes \lambda_{2}+\cdots+K_{i} \lambda_{i} \otimes \lambda_{i}+\cdots+K_{n} \lambda_{n} \otimes \lambda_{n}=\sum_{i=1}^{n} K_{i} \lambda_{i} \otimes \lambda_{i}
$$


(a)
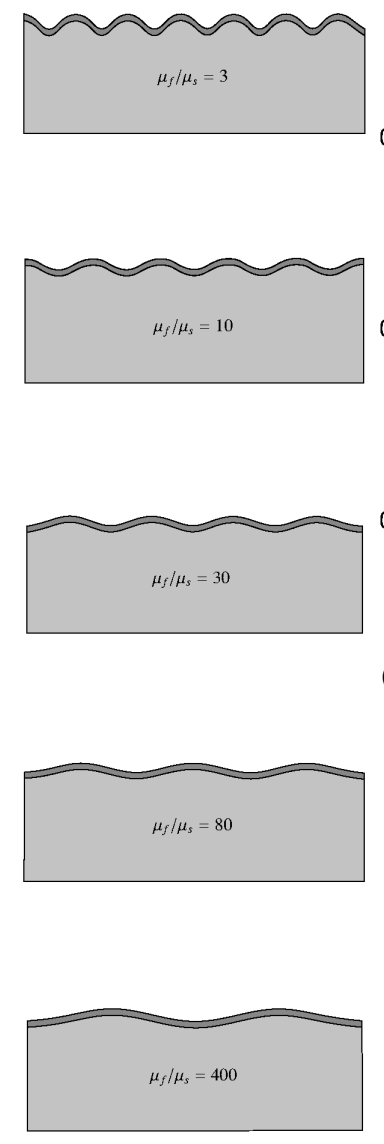

(b)

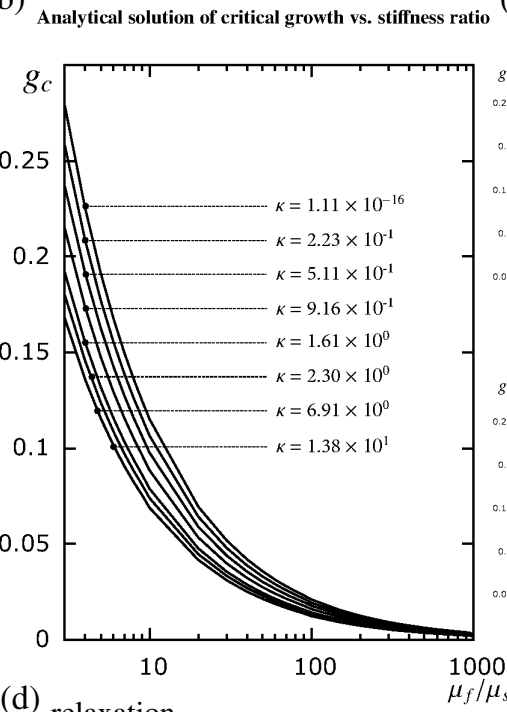

(d) (c)

Analytical vs. Numerical solution of critical growth

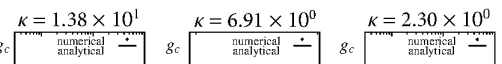

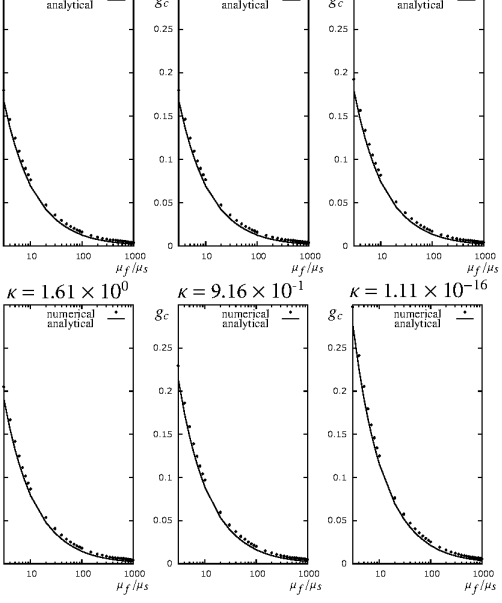

$\kappa$

\begin{tabular}{|c|c|c|}
\hline $7.24 \times 10^{-2}$ & $1.38 \times 10^{1}$ & 0.000001 \\
\hline $1.45 \times 10^{-2}$ & $6.91 \times 10^{0}$ & 0.001 \\
\hline $4.34 \times 10^{-1}$ & $2.30 \times 10^{0}$ & 0.1 \\
\hline $6.21 \times 10^{-1}$ & $1.61 \times 10^{0}$ & 0.2 \\
\hline $1.09 \times 10^{0}$ & $9.16 \times 10^{-1}$ & 0.4 \\
\hline $1.96 \times 10^{0}$ & $5.11 \times 10^{-1}$ & 0.6 \\
\hline $4.48 \times 10^{0}$ & $2.23 \times 10^{-1}$ & 0.8 \\
\hline $9.01 \times 10^{15}$ & $1.11 \times 10^{-16}$ & 1.0 \\
\hline
\end{tabular}

Figure 8. Instability study of growing elastic film with thickness 1 on a viscoelastic substrate by using various relaxation times to examine the viscous substrate effects on the critical growth of the elastic film. The standard solid model is chosen to capture viscoelastic effects. The relation of growth time to relaxation time is $\kappa=\Delta t / \tau$. The stiffness ratio is $\mu_{f} / \mu_{s}$ and the Poisson's ratio for both the film and substrate is $v_{f}=$ $v_{s}=0.45$. The variation of relaxation times occurs by changing the viscosity $\eta$ of the material. This effect could have identical results by holding viscosity constant and changing growth time. The right growing time line shows this parallel impact.

in which $K_{i}$ represents the eigenvalue and $\lambda_{i}$ the associated unit eigenvector for $i=1, \ldots, n$. In the sense of studying growth instability, the negative eigenvalue of stiffness matrices represents the growth instability and the associated unit eigenvector represents the folding pattern. To obtain the critical value of growth, the growth is increased until one of the eigenvalues becomes negative. Then, the associated growth is the critical growth of the system. More computational details are explained in the Appendix.

Figure 8 gathers the analytical results and numerical simulations using eigenvalue analysis. The critical growth $g_{c}$ in Figure 8, b and c, and corresponding folding pattern in Figure 8a are illustrated for different 

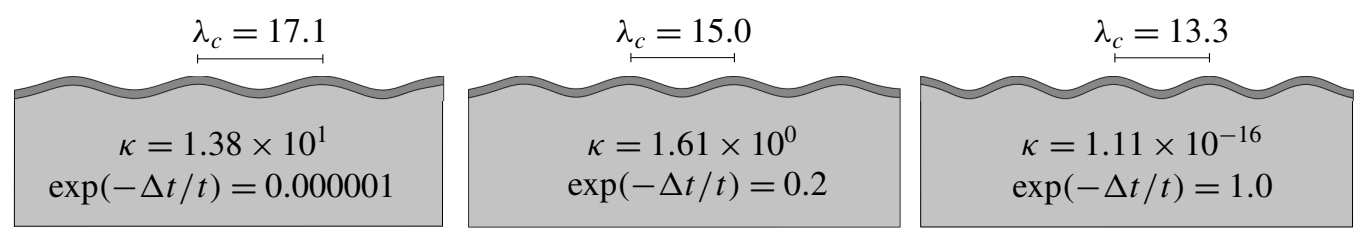

Figure 9. Folding of growing film with thickness 1 on a viscoelastic substrate for some $\kappa=\Delta t / \tau$ with stiffness ratio $\mu_{f} / \mu_{s}=40$. The Poisson's ratio for both the film and substrate is $v_{f}=v_{s}=0.45$. The critical wavelength is denoted $\lambda_{c}=2 \pi / n_{c}$, where the critical wavenumber $n_{c}$ can be calculated from (23).

stiffness ratios $\mu_{f} / \mu_{s}$ for various $\kappa$ in Figure 8d to study the effect of relaxation time on the critical growth $g_{c}$ and wavelength. First, we see that for a given $\tau$ in Figure 8b, the critical growth decreases by increasing the stiffness ratio. Second, it is observed that the critical growth $g_{c}$ increases by decreasing $\kappa$ or alternatively increasing the relaxation time $\tau$. This can be justified by the first observation. That is, for a given $\mu_{f} / \mu_{s}$, a larger relaxation time $\tau$ leads to an increased effective stiffness of the substrate and hence resembles an overall smaller film-to-substrate stiffness ratio which in turn results in a larger critical growth. Third, there is excellent agreement between the numerical results in Figure $8 \mathrm{c}$ using the finite element method via eigenvalue analysis and the proposed analytical solution. So much so that the points corresponding to the numerical results are shown on separate graphs for a better visualization. The numerical results from the finite element method consistently overestimate the analytical solution only and provide an overall stiffer response, as expected. Furthermore, for a given relaxation time, e.g., $\tau=0.0724$, the deformation is illustrated for various stiffness ratios on the left. Increasing the stiffness ratio results in a larger wavelength and thus less waves for a given length of the domain according to (23). Finally, the folding patterns for a given stiffness ratio of $\mu_{f} / \mu_{s}=40$ but for varying relaxation time $\tau$ are illustrated in Figure 9 and it is obvious that increasing the relaxation time decreases the wavelength. This can again be justified by the fact that increasing the relaxation time is effectively decreasing the stiffness ratio and hence the wavelength.

\section{Conclusion}

Biological growth in living systems can lead to geometric instabilities in the form of folding and wrinkling, thus understanding these phenomenon is of crucial importance. Growth-induced instabilities are often studied in bilayer systems where both the thin film and the underlying compliant substrate behave elastically. Nonetheless, due to its relevance for living tissues, the substrate in this contribution is considered to be viscoelastic. This problem is carefully analyzed using both an analytical approach as well as computational simulations using the finite element method whereby eigenvalue analysis is utilized to capture the instabilities. The results obtained from both methods are compared for a wide range of parameters and show an excellent agreement between the computational simulations and the proposed analytical solution. It is observed that the viscoelastic influence of the substrate can be interpreted and eventually replaced by an "effective" elastic model. Our next immediate extension of this contribution is to replace the perfect bonding between the substrate and the film by a general interface model [Javili et al. 2017; Javili 2018] and study its implications. 
read data: geometrical data, material parameters, and boundary conditions

initialization: set degrees of freedom, quadrature points, and shape functions

while eigenvalues $>0$ do

calculate Neumann, Dirichlet, and loads for this time step

while Newton loop do

initialize global tangent stiffness matrix, residuum, volume, surface, and internal forces

for element loop do

determine DOFs, displacement, and coordinates belonging to the current element

for integration loop do

evaluate shape function and its gradient at the current quadrature point

calculate deformation gradient $\boldsymbol{F}$

if film element then

| elasticity material box ( $\boldsymbol{F}$, state variables)

else

| viscoelasticity material box $(\boldsymbol{F}$, state variables $)$

end

for node loop do

I assemble element stiffness matrix $\boldsymbol{K}$

end

end

assemble global stiffness matrix, volume, surface, and internal forces

end

calculate residual $\boldsymbol{r}^{\varphi}(\varphi(X, t))$

end

eigenvalue analysis

if eigenvalue $<0$ then

$g=g_{c r}$

calculate eigenvector

break while loop

else

| growth increment $g_{\text {new }}=g_{\text {old }}+\Delta g$

end

end

Algorithm 1. The incremental nonlinear finite element method with eigenvalue analysis to capture geometrical instabilities.

\section{Appendix: Computational aspects}

The geometry in Figure 7 consists of a rectangular domain which is meshed with 560 quadratic quadrilateral elements with 3578 DOFs. Computations are carried out using our in-house nonlinear finite element code; to have a proper numerical solution we use the finite element method algorithm 1, which explains the finite element structure using eigenvalue analysis. Algorithm 2 is an elastic material box used to model growth of elastic film and Algorithm 3 is a viscoelastic material box to model the substrate behavior. 
Input: deformation gradient $(\boldsymbol{F})$, state variables

Update configurations $J_{n+1}:=\operatorname{det}\left[\boldsymbol{F}_{n+1}\right], \boldsymbol{C}_{n+1}=\boldsymbol{F}_{n+1}^{t} \boldsymbol{F}_{n+1}$

Decompose $\boldsymbol{F}$ to $\boldsymbol{F}_{e}$ and $\boldsymbol{F}_{g}$

Calculate growth part $\boldsymbol{F}_{g}$

Compute $P, \mathbb{C}$

Output: Piola stress and algorithmic tangent moduli

Algorithm 2. Elastic material box to calculate growth in living materials.

Input: deformation gradient $(\boldsymbol{F})$, state variables

Update configurations $\boldsymbol{J}_{n+1}:=\operatorname{det}\left[\boldsymbol{F}_{n+1}\right], \boldsymbol{C}_{n+1}=\boldsymbol{F}_{n+1}^{t} \boldsymbol{F}_{n+1}, \overline{\boldsymbol{F}}_{n+1}=J_{n+1}^{-1 / 3} \boldsymbol{F}_{n+1}, \overline{\boldsymbol{C}}_{n+1}=J_{n+1}^{-2 / 3} \boldsymbol{C}_{n+1}$ calculate the second Piola-Kirchhoff stress $\boldsymbol{S}_{n+1}$ and internal variable $\boldsymbol{Q}_{n+1}$

Compute $P, \mathbb{C}$

Output: Piola stress, algorithmic tangent moduli

Algorithm 3. Viscoelasticity material box to calculate viscoelastic effects of substrate.

\section{References}

[Allen 1969] H. G. Allen, Analysis and design of structural sandwich panels, Pergamon, Oxford, 1969.

[Balbi and Ciarletta 2013] V. Balbi and P. Ciarletta, "Morpho-elasticity of intestinal villi", J. R. Soc. Interface 10:82 (2013), art. id. 20130109.

[Ben Amar and Goriely 2005] M. Ben Amar and A. Goriely, "Growth and instability in elastic tissues", J. Mech. Phys. Solids 53:10 (2005), 2284-2319.

[Budday and Steinmann 2018] S. Budday and P. Steinmann, "On the influence of inhomogeneous stiffness and growth on mechanical instabilities in the developing brain", Int. J. Solids Struct. 132-133 (2018), 31-41.

[Budday et al. 2014] S. Budday, P. Steinmann, and E. Kuhl, "The role of mechanics during brain development", J. Mech. Phys. Solids 72 (2014), 75-92.

[Budday et al. 2015] S. Budday, E. Kuhl, and J. W. Hutchinson, "Period-doubling and period-tripling in growing bilayered systems", Philos. Mag. 95:28-30 (2015), 3208-3224.

[Cao and Hutchinson 2012a] Y. Cao and J. W. Hutchinson, "From wrinkles to creases in elastomers: the instability and imperfection-sensitivity of wrinkling”, Proc. R. Soc. Lond. A 468:2137 (2012), 94-115.

[Cao and Hutchinson 2012b] Y. Cao and J. W. Hutchinson, "Wrinkling phenomena in neo-Hookean film/substrate bilayers", $J$. Appl. Mech. 79:3 (2012), art. id. 031019.

[Ciarletta and Ben Amar 2012] P. Ciarletta and M. Ben Amar, "Growth instabilities and folding in tubular organs: a variational method in non-linear elasticity", Int. J. Non-Linear Mech. 47:2 (2012), 248-257.

[Ciarletta and Maugin 2011] P. Ciarletta and G. A. Maugin, "Elements of a finite strain-gradient thermomechanical theory for material growth and remodeling", Int. J. Non-Linear Mech. 46:10 (2011), 1341-1346.

[Ciarletta et al. 2013] P. Ciarletta, L. Preziosi, and G. A. Maugin, "Mechanobiology of interfacial growth", J. Mech. Phys. Solids 61:3 (2013), 852-872.

[Cowin and Hegedus 1976] S. C. Cowin and D. H. Hegedus, "Bone remodeling, I: Theory of adaptive elasticity", J. Elasticity 6:3 (1976), 313-326.

[Dervaux and Ben Amar 2011] J. Dervaux and M. Ben Amar, "Buckling condensation in constrained growth", J. Mech. Phys. Solids 59:3 (2011), 538-560. 
[Dunlop et al. 2010] J. W. C. Dunlop, F. D. Fischer, E. Gamsjäger, and P. Fratzl, "A theoretical model for tissue growth in confined geometries", J. Mech. Phys. Solids 58:8 (2010), 1073-1087.

[Epstein and Maugin 2000] M. Epstein and G. A. Maugin, "Thermomechanics of volumetric growth in uniform bodies", Int. J. Plast. 16:7-8 (2000), 951-978.

[Garikipati et al. 2004] K. Garikipati, E. M. Arruda, K. Grosh, H. Narayanan, and S. Calve, "A continuum treatment of growth in biological tissue: the coupling of mass transport and mechanics", J. Mech. Phys. Solids 52:7 (2004), 1595-1625.

[Goriely et al. 2008] A. Goriely, M. Robertson-Tessi, M. Tabor, and R. Vandiver, "Elastic growth models", pp. 1-44 in Mathematical modelling of biosystems, edited by R. P. Mondaini and P. M. Pardalos, Appl. Optim. 102, Springer, 2008.

[Holzapfel 2000] G. A. Holzapfel, Nonlinear solid mechanics: a continuum approach for engineering, Wiley, Chichester, England, 2000.

[Hong et al. 2009] W. Hong, X. Zhao, and Z. Suo, "Formation of creases on the surfaces of elastomers and gels", Appl. Phys. Lett. 95:11 (2009), art. id. 111901.

[Huang 2005] R. Huang, "Kinetic wrinkling of an elastic film on a viscoelastic substrate", J. Mech. Phys. Solids 53:1 (2005), $63-89$.

[Huang and Suo 2002] R. Huang and Z. Suo, "Instability of a compressed elastic film on a viscous layer", Int. J. Solids Struct. 39:7 (2002), 1791-1802.

[Huang et al. 2005] Z. Y. Huang, W. Hong, and Z. Suo, "Nonlinear analyses of wrinkles in a film bonded to a compliant substrate", J. Mech. Phys. Solids 53:9 (2005), 2101-2118.

[Hutchinson 2013] J. W. Hutchinson, "The role of nonlinear substrate elasticity in the wrinkling of thin films", Phil. Trans. R. Soc. A 371:1993 (2013), art. id. 20120422.

[Javili 2018] A. Javili, "Variational formulation of generalized interfaces for finite deformation elasticity", Math. Mech. Solids 23:9 (2018), 1303-1322.

[Javili et al. 2013] A. Javili, A. McBride, and P. Steinmann, "Thermomechanics of solids with lower-dimensional energetics: on the importance of surface, interface, and curve structures at the nanoscale", Appl. Mech. Rev. 65:1 (2013), art. id. 010802.

[Javili et al. 2014] A. Javili, P. Steinmann, and E. Kuhl, "A novel strategy to identify the critical conditions for growth-induced instabilities", J. Mech. Behav. Biomed. Mater. 29 (2014), 20-32.

[Javili et al. 2015] A. Javili, B. Dortdivanlioglu, E. Kuhl, and C. Linder, "Computational aspects of growth-induced instabilities through eigenvalue analysis", Comput. Mech. 56:3 (2015), 405-420.

[Javili et al. 2017] A. Javili, P. Steinmann, and J. Mosler, "Micro-to-macro transition accounting for general imperfect interfaces", Comput. Methods Appl. Mech. Eng. 317 (2017), 274-317.

[Jin et al. 2011] L. Jin, S. Cai, and Z. Suo, "Creases in soft tissues generated by growth”, Europhys. Lett. 95:6 (2011), art. id. 64002 .

[Jin et al. 2015] L. Jin, A. Auguste, R. C. Hayward, and Z. Suo, "Bifurcation diagrams for the formation of wrinkles or creases in soft bilayers", J. Appl. Mech. 82:6 (2015), art. id. 061008.

[Kuhl 2014] E. Kuhl, "Growing matter: a review of growth in living systems", J. Mech. Behav. Biomed. Mater. 29 (2014), 529-543.

[Kuhl and Steinmann 2003] E. Kuhl and P. Steinmann, "Mass- and volume-specific views on thermodynamics for open systems", Proc. R. Soc. Lond. A 459:2038 (2003), 2547-2568.

[Kuhl et al. 2003] E. Kuhl, A. Menzel, and P. Steinmann, "Computational modeling of growth", Comput. Mech. 32:1-2 (2003), $71-88$.

[Li et al. 2011] B. Li, Y.-P. Cao, X.-Q. Feng, and H. Gao, "Surface wrinkling of mucosa induced by volumetric growth: theory, simulation and experiment", J. Mech. Phys. Solids 59:4 (2011), 758-774.

[Moulton and Goriely 2011] D. E. Moulton and A. Goriely, "Circumferential buckling instability of a growing cylindrical tube", J. Mech. Phys. Solids 59:3 (2011), 525-537.

[Podio-Guidugli 2000] P. Podio-Guidugli, “A primer in elasticity”, J. Elasticity 58:1 (2000), 1-104.

[Rodriguez et al. 1994] E. K. Rodriguez, A. Hoger, and A. D. McCulloch, "Stress-dependent finite growth in soft elastic tissues", J. Biomech. 27:4 (1994), 455-467. 
[Simo and Hughes 1998] J. C. Simo and T. J. R. Hughes, Computational inelasticity, Interdisc. Applied Math. 7, Springer, 1998.

[Sultan and Boudaoud 2008] E. Sultan and A. Boudaoud, "The buckling of a swollen thin gel layer bound to a compliant substrate", J. Appl. Mech. 75:5 (2008), art. id. 051002.

[Sun et al. 2012] J.-Y. Sun, S. Xia, M.-W. Moon, K. H. Oh, and K.-S. Kim, "Folding wrinkles of a thin stiff layer on a soft substrate", Proc. R. Soc. Lond. A 468:2140 (2012), 932-953.

[Taber 1995] L. A. Taber, "Biomechanics of growth, remodeling, and morphogenesis", Appl. Mech. Rev. 48:8 (1995), 487-545.

[Tepole et al. 2011] A. B. Tepole, C. J. Ploch, J. Wong, A. K. Gosain, and E. Kuhl, "Growing skin: a computational model for skin expansion in reconstructive surgery”, J. Mech. Phys. Solids 59:10 (2011), 2177-2190.

[Wiggs et al. 1997] B. R. Wiggs, C. A. Hrousis, J. M. Drazen, and R. D. Kamm, "On the mechanism of mucosal folding in normal and asthmatic airways", J. Appl. Physiol. 83:6 (1997), 1814-1821.

[Wyczalkowski et al. 2012] M. A. Wyczalkowski, Z. Chen, B. A. Filas, V. D. Varner, and L. A. Taber, "Computational models for mechanics of morphogenesis", Birth Defects Res. C Embryo Today 96:2 (2012), 132-152.

[Xie et al. 2014] W.-H. Xie, B. Li, Y.-P. Cao, and X.-Q. Feng, "Effects of internal pressure and surface tension on the growthinduced wrinkling of mucosae", J. Mech. Behav. Biomed. Mater. 29 (2014), 594-601.

[Xu et al. 2010] G. Xu, A. K. Knutsen, K. Dikranian, C. D. Kroenke, P. V. Bayly, and L. A. Taber, "Axons pull on the brain, but tension does not drive cortical folding”, J. Biomech. Eng. (ASME) 132:7 (2010), art. id. 071013.

[Xu et al. 2014] F. Xu, M. Potier-Ferry, S. Belouettar, and Y. Cong, "3D finite element modeling for instabilities in thin films on soft substrates”, Int. J. Solids Struct. 51:21-22 (2014), 3619-3632.

[Yavari 2010] A. Yavari, “A geometric theory of growth mechanics”, J. Nonlinear Sci. 20:6 (2010), 781-830.

Received 3 Aug 2018. Revised 2 Sep 2018. Accepted 9 Sep 2018.

IMAN VALIZADEH: iman.valizadeh@udo.edu

Department of Mechanical Engineering, TU-Dortmund, Dortmund, Germany

PAUl STEINMANN: paul.steinmann@fau.de

Chair of Applied Mechanics, Universität Erlangen-Nürnberg, Erlangen, Germany

and

Glasgow Computational Engineering Centre, School of Engineering, University of Glasgow, Glasgow, UK

ALI JAVILI: ajavili@bilkent.edu.tr

Department of Mechanical Engineering, Bilkent University, Ankara, Turkey 